\title{
Estrategias docentes que mediaron en el aprendizaje de la lectoescritura en estudiantes con discapacidad múltiple del Centro de Educación Especial Carlos Luis Valle Masís, Costa Rica
}

\author{
Farlin Arias Cerdas ${ }^{1}$ - Lesly Badilla Dover ${ }^{2}$ - Ana María Chinchilla Meza ${ }^{3}$ - \\ Johanna Mata Orozco ${ }^{4}$ - Joyce Orozco Brenes ${ }^{5}$ - María de los Ángeles Carpio Brenes ${ }^{6}$ \\ Universidad de Costa Rica, Costa Rica
}

\section{Resumen}

Recepción: 30-09-2016 / Aceptación: 30-11-2016

Este artículo es el producto de un Seminario de Graduación para optar por la Licenciatura en Educación Especial con énfasis en Discapacidad Múltiple de la Universidad de Costa Rica, cuyo objetivo principal fue analizar las estrategias docentes utilizadas en la enseñanza de la lectoescritura de estudiantes con discapacidad múltiple.

La investigación se orientó en una perspectiva cualitativa con un diseño fenomenológico. Se trabajó con ocho docentes del Centro de Educación Especial Carlos Luis Valle Masis, en Costa Rica, que a lo largo de sus carreras profesionales, habían enseñado a leer y escribir con éxito a estudiantes con esta condición. El análisis de la información se realizó mediante la triangulación al comparar los datos obtenidos de distintas fuentes (ocho docentes), mediante dos técnicas (entrevista y autoreporte), y basadas en la secuencia de acciones a seguir en el análisis fenomenológico de los datos obtenidos en una entrevista propuesta por Mari, Bo y Climent (2010).

Los resultados muestran que para que el estudiantado con discapacidad múltiple pueda acceder al aprendizaje de la lectoescritura, es necesario la presencia de tres condiciones: las características del profesorado, las particularidades del estudiantado y el sistema de apoyos. En lo que respecta a las estrategias, es decir, las acciones que se realizan para el alcance de objetivos del proceso educativo, el profesorado destaca tres aspectos básicos: un diagnóstico exhaustivo, la selección del método a partir de las características del estudiantado y la adaptación de la técnica al sistema de comunicación particular.

Palabras clave: discapacidad múltiple, lectura, escritura, enseñanza, aprendizaje, comunicación alternativa.

\begin{abstract}
This article is the result of a Graduation Seminar to obtain the degree of bachelor in Special Education, focused on Multiple Disabilities of the University of Costa Rica which main objective was to analyze the teaching strategies of literacy acquisition of multi disabled students.

The research was oriented toward a qualitative perspective with a phenomenological design. It was held with the cooperation of eight teachers of the Special Education Center Carlos Luis Valle Masís, in Costa Rica, who along their careers had successfully instructed students, under this condition, in reading and writing. The analysis of the information was triangulated by comparing the data obtained from different sources (eight teachers), by means of two research techniques (interview and self-report), and based on the sequence of actions to be followed in the phenomenological analysis of the data collected in an interview proposed by Mari, Bo and Climent (2010).

The results show that in order for the students with multiple disabilities to have access to literacy acquisition, it is necessary to meet three conditions: the characteristics of the teaching body, the particularities of the students and the support systems. With regard to the strategies, namely, the actions carried out to achieve the objectives of the instructional process, the teaching faculty highlights three basic aspects: a comprehensive diagnosis, the selection of the method based on the characteristics of the students and the adaptation of the technique to the particular communication system.
\end{abstract}

Key Words: multiple disability, reading, writing, teaching, learning, alternative communication.

1. Licenciada en Ciencias de la Educación con énfasis en Discapacidad Múltiple Docente, Escuela República de Corea, Costa Rica;; email: far.arias22@gmail.com 2. Licenciada en Ciencias de la Educación con énfasis en Discapacidad Múltiple Docente Docente, Centro de Educación Especial de Guápiles. Costa Rica; email: lesbdover@gmail.com

3. Licenciada en Ciencias de la Educación con énfasis en Discapacidad Múltiple Docente Docente, Centro de Enseñanza Especial Carlos Luis Valle Masís, Costa Rica; email: anychi85@gmail.com

4. Licenciada en Ciencias de la Educación con énfasis en Discapacidad Múltiple Docente Docente, Centro de Enseñanza Especial Carlos Luis Valle Masís, Costa Rica; email: johamat92@hotmail.com

5. Licenciada en Ciencias de la Educación con énfasis en Discapacidad Múltiple Docente Docente, Centro de Educación Especial de Atención Integral de Goicoechea, Costa Rica; email: joyce.orobre24@gmail.com

6. Doctora en Educación, Investigadora-Docente, Universidad de Costa Rica; email: marycarpiob@gmail.com

ISSN 2311-5513 - Febrero 2017 


\section{Introducción}

El enfoque actual de atención a la diversidad dicta que el curriculum oficial de un país, como referente para el diseño y puesta en marcha de los procesos educativos, debe ser un instrumento para asegurar la igualdad de oportunidades (Duk y Loren, 2010). No obstante, en la atención educativa que recibe la población costarricense con discapacidad múltiple -condición que implica la combinación de dos o más discapacidades, de carácter psíquico, sensorial y físico (Hernández, 2012)dicha premisa resulta una utopía. ¿Por qué?

En Costa Rica, el Ministerio de Educación Pública (MEP), mediante las Normas y Procedimientos para el Manejo de los Servicios Educativos para Estudiantes con Discapacidad Múltiple, enfatiza que la prioridad en la atención de esta población estudiantil debe centrase en el "desarrollo de habilidades funcionales que le den la oportunidad de ser lo más independiente posible, de adquirir habilidades sociales para su desempeño en grupos $\mathrm{y}$ de desenvolverse en ambientes cada vez menos restringidos" (Ministerio de Educación Pública, 2005, p. 41).

Según la normativa, el Programa Individual de Enseñanza (PIE) debe enfocarse en el desarrollo de condiciones de salud, higiene y nutrición básicas para su bienestar; de destrezas funcionales de aprendizaje, y de habilidades adaptativas en general, en ambientes naturales y acordes a la capacidad individual y edad cronológica del estudiantado. En el área del lenguaje, la prioridad es la comunicación alternativa, pero no enfocada hacia el aprendizaje de la lectura y escritura (Ministerio de Educación Pública, 2012).

Las investigaciones realizadas en Costa Rica sobre la enseñanza aprendizaje de esta población estudiantil se orientan precisamente hacia la comunicación alternativa, dejando de lado la lectoescritura. Se encontraron algunos estudios relacionados con las barreras de la comunicación y las estrategias para su abordaje en la población con discapacidad múltiple (Chacón, Dewey, López, Orozco, Salas y Vaglio, 1994; Bermúdez y López, 2001; Mora, 2001); y otros sobre los factores que influyen en el aprendizaje de la lectoescritura y estrategias metodológicas para su enseñanza, pero a poblaciones con otras discapacidades (Fernández, Alvarado y Zúñiga, 1996; Bermúdez 2002; Cantero, 2005; Fonseca, 2005; Cisneros y Kavanaugh, 2006; Leiva y Ruíz, 2008; Vargas y Venegas, 2008).

Teóricos de otros países como Martínez de Morentin, Sanciñena, Sánchez, Sánchez y Yoldi (2000) afirman que el poco abordaje de la lectoescritura en esta población escolar se debe a que:

a un alumno con discapacidad motórica grave se le suele asociar de inmediato una limitada capacidad intelectual. En realidad, en los niños con discapacidad motórica su potencial cognitivo no depende de su afección motora. Este error supone en la práctica unas bajas expectativas del alumno y por lo tanto una disminución en el nivel de exigencia en objetivos y contenidos (p. 79). 
Miranda (2009), destaca que hay estudiantes con discapacidades motoras severas y con habilidades cognitivas conservadas, que usan sistemas alternativos de comunicación que pueden beneficiarse con una intervención en lectoescritura más formal, pero no llega a darse por la poca formación del profesorado, las bajas expectativas familiares y escolares. Por su parte, Erickson, Koopenhaver y Yoder (2002) afirman que las personas que utilizan sistemas de comunicación alternativa con fuertes habilidades lingüísticas expresivas y receptivas, pueden alcanzar altos niveles de alfabetización, por lo que, independientemente de la magnitud o severidad de su discapacidad, tienen el derecho de aprender a leer y escribir.

Estudios recientes demuestran que esta población estudiantil puede acceder a la lectoescritura siempre y cuando cuenten con ciertas destrezas motoras, cognitivas y lingüísticas que se los permitan. Nuñez y Santamaría (2014) afirman que los prerrequisitos fundamentales para iniciar el aprendizaje de la lectoescritura son los procesos cognitivos y las habilidades de la lengua; Cuetos (2008) coincide al señalar como requisitos, los factores lingüísticos y los factores cognitivos.

En el área cognitiva, Moraleda, Romero y Cayetano (2013) afirman que entre un 30\% y un $70 \%$ de niños con parálisis cerebral presentan disfunción cognitiva (2013). Muriel, GarcíaMolina, Aparicio-López, Ensañat y Roig-Rovira (2014) señalan que los déficits cognitivos de la población con parálisis cerebral infantil, como las alteraciones de la atención, visuopercepción, déficits en el funcionamiento ejecutivo y memoria de trabajo, pueden mejorar tras la aplicación de un programa de rehabilitación cognitiva. Un adecuado desarrollo de estas destrezas en el estudiantado con discapacidad múltiple son las que determinarán si el aprendizaje de la lectoescritura es una opción pertinente.

En el componente lingüístico, es indispensable valorar tempranamente la competencia comunicativa para ver la posibilidad del uso de un sistema alternativo, pues como afirma el Ministerio de Educación Pública (2012) se parte de la premisa de que todas las personas pueden llegar a comunicarse de una $\mathrm{u}$ otra manera. La comunicación alternativa se refiere "a las formas de comunicación distintas del habla, que ayudan a potenciarla o a sustituirla cuando el sujeto tiene grave compromiso para comunicarse como lo hace el común de la gente" (Toro, 2008, p. 65).

Con respecto al área motriz, es necesario determinar en qué medida el cuadro motor del estudiante con discapacidad múltiple posibilita el uso funcional de sus manos: el tono muscular, fuerza y la intencionalidad manipulativa de éstas, cuál de ellas utiliza preferentemente, habilidad para sostener y soltar objetos, si sostiene por momentos prolongados, si puede desplazar piezas por arrastre, el tipo de prensa que realiza (esférica, cilíndrica, pinza), así como la capacidad que tiene para señalar. A nivel de tronco y cabeza, es indispensable valorar el grado de control de 
los mismos y si puede mantener una postura sentada al hacer actividades con las manos. Esta información permitirá hacer las adaptaciones de mobiliario escolar y de material didáctico que requiere cada estudiante (Consejo Nacional de Fomento Educativo, 2010).

Ahora bien, no es suficiente que el estudiantado con discapacidad múltiple cumpla con los requisitos para acceder al aprendizaje de la lectoescritura, sino que se haga efectiva su enseñanza, pues "las dimensiones epistémicas (la cualidad de los conocimientos que provienen de las ciencias, para realizar la adecuada transposición didáctica) y metodológicas (las estrategias de enseñanza) como garantes del acceso a la alfabetización, resultan de un valor incalculable." (Grzona, 2015, p. 119).

Montes de Oca y Machado (2011) señalan cuatro aspectos fundamentales que el profesorado debe considerar en los procesos educativos: las acciones de enseñanza dependen del aprendizaje y guían la actividad del estudiante hacia el alcance de los objetivos; los métodos que se escogen buscan propiciar las capacidades del estudiantado; la actitud positiva hacia el aprendizaje debe ser fomentado en todo momento; y el respeto por la autonomía del estudiante es el eje primordial. Grzona (2015) destaca dos premisas básicas para el abordaje de la población con discapacidad múltiple: se debe ofrecer los formatos accesibles a partir del análisis minucioso de las formas de comunicación que cada estudiante necesite y la funcionalidad que le permita actuar con la mayor independencia en su entorno.
Con respecto al método de enseñanza de la lectoescritura más conveniente para esta población, Ramos (2004) afirma que "no existen métodos de lectura eficaces sólo para un determinado tipo de alumnos con discapacidad, o al menos, consideramos que si el procedimiento es adecuado para el alumno con discapacidad, también lo puede ser para el resto" (p. 201). Le corresponde al profesorado escoger, de entre una variedad de propuestas, la que mejor se ajuste a las características y necesidades de sus estudiantes, partiendo de las técnicas, actividades y materiales que cada uno de los métodos propone.

Sin lugar a dudas, como afirma Steinbach (2016), el estudiantado con discapacidad múltiple tiene derecho a acceder a una instrucción integral de alfabetización, pero para hacerlo efectivo, necesitan docentes que les brinde los apoyos apropiados, que cuenten con la capacitación suficiente para enseñarles, pero especialmente, que tengan actitud para hacerlo, pues de sus decisiones dependerá la superación de las barreras de accesibilidad en el ámbito escolar.

Esta no es una tarea fácil para el profesorado, pues a cada estudiante con discapacidad múltiple debe diseñarle una estrategia, es decir, debe hacer "un procedimiento heurístico que permite tomar decisiones en condiciones específicas" (Latorre y Seco, 2013, p.15); en este caso, relacionadas con la enseñanza-aprendizaje de la lectoescritura.

El estudio que se reporta aquí tiene que ver con esto, pues se realizó con dos objetivos 
principales: identificar las condiciones que mediaron en el aprendizaje de la lectoescritura de estudiantes con discapacidad múltiple del Centro de Educación Especial Carlos Luis Valle Masís, en Costa Rica; y conocer las estrategias que utilizó el profesorado de Educación Especial de esa institución para enseñar esta habilidad a esa población escolar.

\section{Metodología}

Esta investigación se enmarcó en un enfoque cualitativo, pues de acuerdo con Hernández, Fernández y Baptista (2014), los de este tipo "se fundamentan en una perspectiva interpretativa centrada en el entendimiento del significado de las acciones de las personas" (p. 9). En este caso se esperaba comprender el fenómeno vivido por docentes de Educación Especial que, a lo largo de sus carreras profesionales enseñaron a leer y escribir a estudiantes con discapacidad múltiple, mediante la interpretación de las acciones concretas y conscientes que realizaron en el proceso de enseñanza-aprendizaje para el logro de estas habilidades.

El diseño escogido para el estudio fue el fenomenológico porque, como lo describen los mismos autores, primero se identifica el fenómeno, luego se recopilan los datos de las personas que lo experimentaron, para finalmente desarrollar una descripción compartida de sus experiencias, de lo que vivieron y de qué forma lo hicieron.

El estudio se realizó en Cartago, provincia de Costa Rica, en el Centro de Educación Especial Carlos Luis Valle Masís, institución conformada por 69 docentes de Educación Especial, de los cuales 16 pertenecían al departamento de Discapacidad Múltiple.

Como criterio de selección de las personas participantes, se determinó que sería el profesorado quien contestara de forma afirmativa a la pregunta: ¿ Ha enseñado a leer y escribir de forma efectiva a estudiantes con discapacidad múltiple durante su carrera profesional? El resultado fue de ocho maestras que lo habían hecho, por lo que esa es la cantidad de participantes considerada en este estudio. Su perfil profesional se describe en los resultados.

Las técnicas e instrumentos se seleccionaron a partir de los dos objetivos principales propuestos para la investigación. De esta forma, para identificar las condiciones que mediaron en el aprendizaje de la lectoescritura en estudiantes con discapacidad múltiple, se utilizó la entrevista estructurada con guía de un cuestionario de 11 preguntas (abiertas y cerradas) que solicitaban información al respecto.

Para describir las estrategias docentes utilizadas en la enseñanza de la lectoescritura se utilizó el autoreporte, que "es una técnica donde el mismo informante reportará una situación de su experiencia, solicitada por el investigador" (Alfonzo, 2012, p. 1). A partir de una serie de preguntas, el profesorado describió las técnicas, procedimientos, actividades y materiales que utilizaron en el proceso de enseñanza aprendizaje.

Tanto el cuestionario como la guía del autoreporte, fueron validados por cinco especia- 
listas en Educación Especial, considerados expertos en uno o los dos temas principales de esta investigación: discapacidad múltiple y lectoescritura.

La investigación implicó un proceso de un año, desde febrero del 2015 cuando se realizó la primera reunión con la directora de la institución hasta febrero del 2016, cuando se le entregó el documento con las recomendaciones al profesorado participante. Entre ese lapso se realizaron actividades como las siguientes:

- Reunión con la directora del centro educativo para obtener su autorización y apoyo con el proyecto.

- Elaboración de los instrumentos (cuestionario de la entrevista y la guía de autoreporte) y su respectiva validación.

- Consulta al profesorado del departamento de discapacidad múltiple sobre quienes habían realizado procesos de enseñanza de la lectura y escritura en población con esa condición.

- Entrevistas individuales, con citas previamente establecidas, y con la participación de dos investigadoras para la recopilación de los datos. Cada una de las entrevistas fue grabada en audio, con la autorización de la directora y de cada una de las participantes.

- Charla a las docentes participantes sobre cómo sistematizar, con la técnica del autoreporte, las estrategias que ellas implementaron y que resultaron exitosas en el aprendizaje de la lectura y escritura en sus estudiantes con discapacidad múltiple.
- Plazo de dos meses para hacer el autoreporte de manera escrita; sin embargo, el proceso se extendió por un mes más, porque las labores propias del quehacer escolar impidieron la entrega puntual de este requisito.

- Transcripción de cada una de estas entrevistas y autoreportes, e incorporación en el programa Atlas.ti.

- Análisis de la información a partir de las categorías extraídas del programa Atlas.ti.

- Elaboración de la Memoria de Seminario de Graduación para optar por el título de Licenciatura en Educación Especial con énfasis en Discapacidad Múltiple.

- Devolución de los resultados de la investigación al profesorado participante.

Como el objeto de estudio de la fenomenología "no es ni el sujeto ni el mundo, sino el mundo vivido por el sujeto (...) Y nuestra finalidad, al igual que la de la fenomenología, es la preocupación por la descripción del fenómeno y no su explicación, despreocupándonos de las relaciones causales. El interés estará en mostrar, no en demostrar" (Mari, Bo y Climent, 2010, p.115); es que se escogió el análisis temático para la interpretación de los datos.

La información proporcionada por estas docentes fue grabada, posteriormente transcrita, y después incorporada en el programa Atlas.ti. Como procedimiento para garantizar la interpretación consistente y válida de la in- 
formación obtenida en este análisis temático se recurrió a la triangulación, al comparar y analizar los datos obtenidos de distintas fuentes (las ocho docentes participantes), mediante dos técnicas diferentes (la entrevista y el autoreporte), y basadas en la teoría, tomando como fundamento la propuesta de Mari, Bo y Climent (2010) denominada Secuencia de acciones a seguir en el análisis fenomenológico de los datos obtenidos en una entrevista.

Esta secuencia consiste en los siguientes cinco pasos para la estructuración, análisis e interpretación de toda la información como un conjunto:

- Transcripción de las entrevistas que fueron grabadas con el consentimiento del grupo participante, incluyendo las palabras literales, los incidentes ocurridos y la comunicación no verbal.

- Agrupamiento de las diversas intervenciones en porciones que forman una unidad de significado general que tiene relación directa con el tema de investigación.

- Selección de las unidades generales de significado que son relevantes o no, para construir las categorías temáticas.

- Búsqueda de los criterios que permitan organizar esas unidades en grupos de significado desde los cuales se interpretarán los temas que muestran el fenómeno que vive el grupo participante.

- Realización de un proceso de análisis comprensivo, que busca transformar la vivencia humana en conocimiento sig- nificativo, con los datos personales, palabras literales, estado de ánimo, aspectos no verbales, mención de las categorías y comentarios, que permitan mejorar la comprensión del fenómeno para actuaciones futuras.

\section{Resultados}

De la información proporcionada por el profesorado del Centro de Educación Especial Carlos Luis Valle Masís que ha enseñado a leer y escribir a estudiantes con discapacidad múltiple a lo largo de sus carreras profesionales, se pudo obtener que para que esta población pueda acceder a la lectoescritura, es necesario contar con ciertas condiciones para su aprendizaje y determinadas estrategias para su enseñanza.

\section{Condiciones que mediaron en el aprendi- zaje de la lectoescritura en estudiantes con discapacidad múltiple}

De los datos aportados por las docentes, surgieron tres categorías que podrían considerarse como condiciones que mediaron en el aprendizaje de la lectoescritura: características del profesorado, particularidades del estudiantado y sistema de apoyos.

\section{a. Características del profesorado}

Sin lugar a dudas, la formación del profesorado es uno de los pilares básicos sobre los que se sustenta la calidad de la educación (García, 1999), esto se evidencia en el perfil profesional de las docentes participantes en este estudio, quienes comparten tres elementos en común: formación, experiencia y capacitación. 
- Formación. Las ocho docentes son licenciadas en Educación Especial, seis de ellas de la Universidad de Costa Rica. Tres obtuvieron la especialidad en discapacidad múltiple en esa institución; otras tres, obtuvieron especialidades diferentes dentro de la misma Universidad. Las dos docentes restantes obtuvieron su licenciatura en diversas especialidades de universidades privadas.

- Experiencia. Estas docentes tienen una amplia experiencia laboral que oscila entre 3 y 20 años. Tres enseñaron a leer pero no a escribir, alegando que por las características motoras de sus estudiantes y la carencia de tecnología, tuvieron que recurrir a la lectura por rastreo visual. Otras dos enseñaron solo a escribir porque sus estudiantes habían adquirido la lectura en otro momento. Las tres restantes enseñaron ambas destrezas simultáneamente. La cantidad de estudiantes que cada una ha instruido en lectoescritura a lo largo de sus carreras está entre 1 y 10.

- Capacitación. Por el poco abordaje de este tema en el ámbito costarricense, las docentes afirman haber recurrido a la experimentación, ajustando la metodología de enseñanza de la lectoescritura convencional a esta población; a la pasantía, asistiendo a las clases de otras colegas; y la asesoría, consultando a profesionales conocedores del tema. Actualmente, el profesorado de nuevo ingreso recibe capacitación por parte de las docentes que tienen experiencia en esta área.

\section{b. Particularidades del estudiantado}

Las docentes consideran que para iniciar el proceso de aprendizaje de la lectoescritura, es indispensable identificar, mediante la interacción diaria con el estudiantado, la presencia de dos habilidades básicas: la cognitiva y la lingüística.

- Habilidades cognitivas. Como el estudiantado con discapacidad múltiple no necesariamente tiene comprometido el área intelectual, deben mostrar desarrollo de las destrezas perceptuales como: conocimiento del esquema corporal, manejo de los conceptos espacio-temporales, discriminación visual y auditiva y memoria a corto plazo. De igual forma, debe seguir instrucciones, prestar atención, mantener la concentración en las tareas que se asignan, y especialmente, demostrar motivación, pues no es suficiente saber que puede aprender a leer y escribir, sino estar seguros de que quiere hacerlo.

- Factores lingüísticos. Se refiere a la intencionalidad comunicativa que muestre el o la estudiante, es decir, al deseo de expresar sus ideas de alguna forma. Esta se puede reflejar mediante una mirada, la alteración en el tono muscular, la aceleración del ritmo en la respiración, sudoración, cambios en la postura, la emisión de sonidos, determinados gestos, risas, entre otros.

Las docentes indican que antes de iniciar cualquier proceso de enseñanza, primero hay que identificar el punto de acceso que cada estudiante con discapacidad múltiple utiliza. 
Deliyore (2013), lo define como la parte del cuerpo con la que la persona quiere señalar las opciones que se le proponen. Este puede ser directo, mediante el uso de manos, dedos, pies, codos, rodillas; o indirecto, con el apoyo de punteros cefálicos o teclados virtuales. Luego, se establece un sistema sencillo de comunicación con el uso del sí y no para contestar. Finalmente, se emplean tableros pictográficos grandes para desarrollar el rastreo visual, la precisión y permanencia en el señalamiento con el punto de acceso que utiliza cada estudiante en particular.

\section{c. Sistema de apoyos}

Se refiere al conjunto de recursos materiales y humanos que se encuentran a disposición de la persona con discapacidad para alcanzar su salud y bienestar (Verdugo, 2010). Las docentes reportan el uso de ambos tipos de recursos: material y humano.

- Recurso material. En este estudio se identificaron dos tipos de materiales: el tecnológico y el didáctico. En los materiales tecnológicos se mencionan los de baja tecnología, que son los derivados de la adaptación de objetos simples (CarpioBrenes, 2012), entre los que se destacan el ajuste de crayolas y lápices grandes para la señalización en aquellos estudiantes que podían agarrar y controlar alguna de sus manos; los punteros hechizos con elásticos y jeringas o con varilla de cobre, gorras con varillas de cobre o punteros cefálicos para quienes tenían solo control de cabeza. Los materiales de mediana tec- nología, que corresponde a aparatos más complejos como los pulsadores, las computadoras, el mouse adaptado y las tabletas, se utilizaron con el estudiantado que tenía disociación en los dedos y coordinación viso-motora.

- Entre los recursos didácticos mencionados por las docentes están: las pizarras magnéticas con letras de imán para que puedan deslizarlas el estudiantado que controla movimientos; cartón grande, grueso y con velcro, para el estudiantado con movimientos atetósicos; hojas muy grandes y tableros de deletreo para la escritura por señalamiento manual o cefálica con puntero; cajas de arena o crema de afeitar para seguir trazos sobre la mesa; letras marcadas en tablillas para seguimiento con crayola gruesa.

- Talento humano. Para las docentes entrevistadas, la participación de la familia es esencial, pues el apoyo y el refuerzo que se pueda brindar en la casa es el complemento óptimo para alcanzar los objetivos programados. Sin embargo, primero se debe convencer al padre y la madre de que su hijo o hija puede aprender a leer y escribir, ya que muchas veces se tienen bajas expectativas en esta área por sus características motoras y lingüísticas. Una vez logrado esto, se les imparte talleres donde se elabora material y se les explica la forma de usarlo para reforzar en casa los contenidos de lectoescritura que se van trabajando en clase. 
Estrategias docentes utilizadas en la enseñanza de la lectoescritura de estudiantes con discapacidad múltiple

El profesorado participante en la investigación coincide en que el aprendizaje de la lectoescritura en la población con discapacidad múltiple se da de forma similar al del estudiantado promedio, lo que varía son las estrategias docentes para hacer accesible su enseñanza. Se entiende por estrategia docente "el empleo consciente, reflexivo y regulativo de acciones que se conciben para alcanzar los objetivos del proceso docente-educativo" (Montes de Oca y Machado, 2011, p.5).

De la descripción que las maestras hicieron en sus autoreportes, sobre el procedimiento que siguieron para enseñar a leer y escribir a sus estudiantes con discapacidad múltiple, se pudo extraer tres acciones generales en común: una evaluación diagnóstica detallada, la selección del método a partir de las características del estudiantado y la adaptación de la técnica a cada sistema de comunicación en particular.

\section{a. Evaluación diagnóstica exhaustiva}

La heterogeneidad de la población escolar con discapacidad múltiple exige que se realice un exhaustivo proceso diagnóstico a cada estudiante, para identificar sus características y necesidades en las áreas motora, sensorial, conductual, cognitiva, comunicativa y socioafectiva, y así establecer los apoyos que requiere para acceder al aprendizaje en general.
En lo que respecta a la lectoescritura en particular, las docentes participantes coinciden en que es indispensable determinar cuáles estudiantes tienen las condiciones para generar una forma alternativa de comunicación a través de la lectoescritura. Estas condiciones se refieren a las habilidades cognitivas, las destrezas lingüísticas y el sistema de apoyos para su implementación, que se expusieron anteriormente.

Insisten en la evaluación detallada de las características motoras, pues de esta depende la selección del sistema alternativo de escritura. Mencionan los siguientes aspectos como necesarios de analizar: el tono muscular, los movimientos voluntarios que realiza en cada una de las partes del cuerpo, el señalamiento con alguna parte del cuerpo, el alcance $y$ manipulación de objetos, la forma de agarre, si se sienta solo con buen control de tronco, entre otros.

\section{b. Selección del método apropiado}

De acuerdo con el profesorado participante, no existe una metodología específica para la enseñanza de la lectoescritura a estudiantes con discapacidad múltiple, sino que cualquiera de los métodos que se usa con el estudiantado promedio puede utilizarse también con esta población.

Las docentes señalan que para seleccionar el método apropiado para cada uno de sus estudiantes, consideraron su estilo de aprendizaje (visual, auditivo y kinestésico), pues para quienes son más auditivos el método fonético 
les favorece, mientras que para los que son visuales, el silábico puede resultarles mejor. También tomaron en cuenta el sistema de comunicación alternativa que cada uno de sus estudiantes utilizaba, para ver cuál de los métodos se ajustaba mejor a sus particularidades.

\section{c. Adaptación de la técnica}

La técnica es entendida como un procedimiento lógico, una secuencia de acciones orientadas a obtener unos objetivos propuestos (Ramírez, 2012). Cada método de lectoescritura sugiere una técnica en particular, con determinados pasos, actividades y materiales. Las docentes participantes afirman que siguieron los pasos que establece el método de lectoescritura seleccionado para cada estudiante, pero que fue indispensable adaptar los materiales y modificar las actividades al sistema de comunicación alternativo que utilizaba cada uno de ellos y ellas.

Entre los ajustes que realizaron al material se destacan: la presentación de grafemas y sílabas en tarjetas grandes, de diferentes colores y variedad de texturas; la fusión silábica mediante letras con imán en pizarra magnética; el uso de fichas grandes para formar palabras y oraciones; mostrar objetos concretos o imágenes para ilustrar palabras en estudio.

La adaptación de las actividades incluyó el planteamiento de preguntas cerradas para que las respuestas se limitaran a un sí o no; presentar los textos con letra ampliada, en rotafolios; reforzar la escritura por señalamiento mediante dictados $\mathrm{y}$ formación de palabras, frases y oraciones en fichas que se pudieran deslizar o pegar fácilmente.

A partir de estos hallazgos, el grupo investigador diseñó una guía denominada Lectoescritura en estudiantes con discapacidad múltiple: recomendaciones para el proceso de enseñanza-aprendizaje, donde se ofrecen una serie de sugerencias, organizadas en las tres fases que corresponden a los momentos clave del proceso educativo (diagnóstico, enseñanza y seguimiento), con el propósito de apoyar al profesorado que se inicia en la enseñanza de la lectura y escritura a esta población escolar.

\section{Discusión}

La enseñanza de la lectoescritura en estudiantes con discapacidad múltiple es un tema que ha sido poco abordado en el quehacer pedagógico del profesorado de Educación Especial en Costa Rica. Sin embargo, los teóricos actuales afirman que se le debe ofrecer una respuesta educativa a esta población estudiantil que incluya un sistema de comunicación alternativa que le permita, no solo un desempeño funcional, sino el acceso al currículum académico mediante el aprendizaje de la lectoescritura, con un adecuado sistema de apoyos.

De acuerdo con el profesorado del Centro de Educación Especial Carlos Luis Valle Masís, para que el estudiantado con discapacidad múltiple pueda acceder a la lectoescritura como sistema alternativo de comunicación, es necesario que se den dos aspectos fundamentales: condiciones para el aprendizaje y estrategias para la enseñanza. 
Con relación a las condiciones para el aprendizaje, las docentes destacan tres:

- Características del profesorado, que incluye un perfil docente con tres elementos básicos: formación, experiencia y capacitación.

- Particularidades del estudiantado, enfocadas en la presencia de tres requisitos para la adquisición de la lectoescritura: habilidades cognitivas, destrezas lingüísticas y peculiaridades motoras.

- Sistema de apoyos, relacionados con los recursos materiales (tecnológicos y didácticos) y humanos (familia) con que cuenta cada estudiante en particular.

Con respecto a las estrategias para la enseñanza, también son tres los aspectos resaltan:

- Diagnóstico exhaustivo. Se debe realizar una evaluación detallada y minuciosa de cada estudiante, para conocer las destrezas, intereses, necesidades y potencialidades particulares, especialmente las relacionadas con el aprendizaje de la lectoescritura: habilidades cognitivas, lingüísticas, motoras y los apoyos con que cuentan y los que requieren.
- Selección del método. El proceso de aprendizaje de la lectoescritura en estudiantes con discapacidad múltiple es el mismo que realiza el estudiante promedio, la diferencia radica en el sistema de comunicación, por lo que hay que escoger el método que mejor se ajuste a sus características particulares.

- Adaptación de la técnica. Cada método de lectoescritura sugiere una técnica en particular con una serie de pasos, actividades y materiales. Con la población con discapacidad múltiple se puede seguir la secuencia de acciones tal y como lo propone el método escogido, pero las actividades y materiales deben adaptarse a las destrezas y necesidades de cada estudiante.

Es evidente que el estudiantado con discapacidad múltiple que tiene las condiciones para el aprendizaje de la lectoescritura tiene el derecho de hacerlo, y el profesorado que tiene a cargo su educación, tiene la obligación de apoyarlo con las estrategias docentes pertinentes para su enseñanza.

\section{Referencias}

Alfonzo, N. (2012). Técnicas e Instrumentos de Recolección de Datos Cualitativos. Recuperado de http://www.monografias.com/trabajos93/tecnicas-e-instrumentos-recoleccion-datos-cualitativos/tecnicas-e-instrumentos-recoleccion-datos-cualitativos.shtml

Bermúdez, C. (2002). Comunicación en personas con Discapacidad múltiple. Revista Espiga, 3(6), 23-34. 
Bermúdez, C. y López, W. (2001). Propuesta de intervención pedagógica para el desarrollo de destrezas comunicativas en dos niños con Parálisis Cerebral Infantil. (Tesis de Licenciatura). Universidad de Costa Rica, San José, Costa Rica.

Cantero, R. (2005). Los efectos de la discapacidad mental, sensorial y de comunicación que limitan las funciones cognitivas responsables del aprendizaje de la lectoescritura con el método silábico valoradas mediante el programa de enriquecimiento instrumental de Reuven Feusertein. (Tesis de maestría). Universidad Estatal a Distancia, San José, Costa Rica.

Carpio-Brenes, M. (2012). La Tecnología Asistida como disciplina para la atención pedagógica de personas con discapacidad intelectual. Revista Actualidades Investigativas en Educación Universidad de Costa Rica. (12)2, 1-27

Chacón, D., Dewey, M., López, H., Orozco, J., Salas, M. y Vaglio, V. (1994). Entrenamiento inicial en comunicación funcional por medio de una tabla de comunicación para niños y adolescentes con Discapacidad múltiple y Severa, utilizando el "time delay" y el sistema de procedimientos de instigación gradual (Tesis de Licenciatura). Universidad de Costa Rica, San José, Costa Rica.

Cisneros, D. y Kavanaugh, A. (2006). Herramienta digital para apoyar el proceso de lectoescritura en estudiantes con discapacidad cognitiva. (Tesis de licenciatura). Universidad Nacional de Costa Rica, Heredia, Costa Rica.

Costa Rica, Ministerio de Educación Pública. (2005). Normas y Procedimientos para el Manejo Técnico Administrativo de los Servicios Educativos para Estudiantes con Discapacidad múltiple. San José, Costa Rica: Litografía e Imprenta LIL, S. A.

Costa Rica, Ministerio de Educación Pública. (2012). Orientaciones para el abordaje de los sistemas de comunicación alternativa. Costa Rica: Dirección de Desarrollo Curricular.

Cuetos, F. (2008). Psicología de la escritura. España: Wolters Kluwer España S.A.

Deliyore, R. (2013). Material del curso de la Licenciatura en Educación Especial con énfasis en Discapacidad Múltiple de la Universidad de Costa Rica: Sistemas de comunicación Aumentativa y Alternativa para personas con Discapacidad Múltiple. Universidad de Costa Rica, San José, C.R.

Duk, C., Loren, C. (2010) Flexibilización de currículum para atender la diversidad. Revista Latinoamericana de Educación Inclusiva. 14(1), 187-210.

Erickson, A., Koppenhaver, A. y Yoder, D. (2002). Comunicación Aumentativa: Leer y Escribir. Recuperado de http://www.ceapat.es/InterPresent2/groups/imserso/documents/binario/olasdepalabras.pdf 
Fernández, E., Alvarado, J. y Zúñiga, G. (1996). Análisis de los métodos de lectura y escritura utilizados en las aulas integradas de retraso mental del circuito 05 de la Dirección Regional de Enseñanza de Heredia. (Tesis de licenciatura). Universidad Nacional de Costa Rica, Heredia, Costa Rica.

Fonseca, N. (2005). Valoración de las Ayudas Técnicas Utilizadas en la Alfabetización de las Personas con Discapacidad Visual. (Tesis de maestría). Universidad Estatal a Distancia, San José, Costa Rica.

García, J. (1999). Formación del profesorado. Necesidades y demandas. Barcelona, España. Revista de Educación: Sistemas Nacionales de Evaluación, (321), 454-460.

Grzona, M. (2015). Alfabetización para estudiantes con multidiscapacidad. El desafío de la accesibilidad. En A. Ocampo (Coord.), Lectura para todos. El aporte de la fácil lectura como vía para la equiparación de oportunidades (pp. 117-125). Santiago de Chile: Asociación Española de Comprensión Lectora y Centro de Estudios Latinoamericanos de Educación Inclusiva.

Hernández, M. (2012). El aporte de las madres en los procesos pedagógicos desarrollados en el aula para mejorar la comunicación en el hogar de niños y niñas con retos múltiples del servicio de preescolar del grupo de Discapacidad múltiple del Centro de Enseñanza Especial de San Ramón en el año 2011 (Tesis de Licenciatura). Universidad Latina, Heredia, Costa Rica.

Hernández, R., Fernández, C. y Baptista, M. (2014). Metodología de la investigación. México: Mc Graw Hill Education.

Latorre, M. y Seco, C. (2003). Estrategias y técnicas metodológicas. Perú: Universidad Marcelino Chanpagnat.

Leiva, E. y Ruiz, R. (2008). Propuesta de estrategias metodológicas en el área de lectoescritura, para estudiantes del nivel de III Ciclo de Educación Especial del Colegio Técnico Profesional de la Fortuna de Bagaces durante el III trimestre del curso lectivo 2007. (Tesis de maestría). Universidad Estatal a Distancia, Liberia, Costa Rica.

Mari, R., Bo, R. y Climent, C. (2010). Propuesta de análisis fenomenológico de los datos obtenidos en la entrevista. Revista de Ciències de l’Educació, 1, 113-133.

Martínez de Morentin, M., Sanciñena, M., Sánchez, M., Sánchez, C., Yoldi, G. (2000). Necesidades Educativa Especiales: alumnado con discapacidad motórica; Guía para la respuesta educativa a las necesidades del alumnado con Parálisis Cerebral. España: Gobierno de Navarra, Departamento de Educación y Cultura. 
México, Consejo Nacional de Fomento Educativo (2010). Discapacidad motriz. Guía didáctica para la inclusión en educación inicial y básica.

Miranda, E. (2009). Lectoescritura para todos. España: Ministerio de Educación y Ciencia.

Montes de Oca, N. y Machado, E. (2011). Estrategias docentes y métodos de enseñanza-aprendizaje en la Educación Superior. Humanidades Médicas, 11(3), 475-488.

Mora, D. (2001). Efectos de la músico terapia y la danza terapia en el comportamiento no verbal de las personas con discapacidad múltiple severa (Tesis de licenciatura). Universidad de Costa Rica, San José, C.R.

Moraleda, E., Romero, M. y Cayetano, M. (2013). La parálisis cerebral como una condición dinámica del cerebro: un estudio secuencial del desarrollo de niños hasta los 6 años de edad. Universitas Psychologica, (12)1, 119-127.

Muriel, V., García-Molina, A., Aparicio-López, C., Ensañat, A. y Roig-Rovira, T. (2014). Estimulación cognitiva en niños con parálisis cerebral. Revista de Neurología. (59)10, 443-448.

Nuñez, M. y Santamarina, M. (2014). Prerrequisitos para el proceso de aprendizaje de la lectura y la escritura: conciencia fonológica y destrezas orales de la lengua. Revista Lengua y Habla; Revista del Centro de Investigación y Atención Lingüística C.I.A.L., (18), 72-92.

Ramírez, L. (s.f). Necesidad de conocer por parte del docente sobre la aplicación de las adecuaciones curriculares en el aula a un estudiante con discapacidad de tetraplejia. (Tesis de licenciatura). Universidad Estatal a Distancia, Heredia, Costa Rica.

Steinbach, D. (2016). The Right to Write: Even if I Can't Hold a Pencil. Recuperado de https ://91372e5fba0d1fb26b7213cee80c2bfb23b1a8fcedea15638c1f.ssl.cf1.rackcdn.com/materials/ ihavetherighttowritechapter1_Jan_9_2016-21_00_03.pdf

Toro, I. (2008). Comunicación, lenguaje y tecnología para la inclusión educativa. Bogotá, Colombia: Universidad Pedagógica Nacional.

Verdugo, M. (2010). Discapacidad Intelectual. Definición, clasificación y sistemas de apoyo. España: Editorial Alianza.

Vargas, M. y Venegas, J. (2008). Sistematización de una experiencia de alfabetización de acuerdo con las potencialidades y necesidades de una persona adulta no alfabetizada con discapacidad cognitiva. (Tesis de licenciatura). Universidad Nacional de Costa Rica, Heredia, C.R. 